\title{
Vigilância sanitária: revisão integrativa dos processos de capacitação da força de trabalho
}

\author{
Marcela Bella Lopes \\ Graduação em Farmácia (2001) e Farmácia e Bioquímica (2002) pela Universidade Federal de Juiz de Fora. Pós- \\ graduação lato sensu em Farmacologia: Atualização e Novas Perspectivas pela Universidade Federal de Lavras \\ (2005) e pós-graduação lato sensu em Gestão de Saúde Pública pela Faculdade Finon (2010). Cursando mestrado \\ profissional na Universidade Federal Fluminense em Ensino na Saúde. \\ $\triangle$ bellalps@hotmail.com
}

\section{Benedito Carlos Cordeiro}

Graduação em Farmácia pela Universidade Federal de Santa Catarina (1984), graduação em Direito pela Universidade do Vale do Itajaí (1993), mestrado em Saúde Pública pela Universidade de São Paulo (2001) e doutorado em Saúde Pública pela Universidade de São Paulo (2008).

\begin{abstract}
Resumo:
Evidências de estudo de Vigilância Sanitária (Visa) têm demonstrado a falta de financiamento para executar as ações de Visa, consequentemente, com poucas atividades de capacitação na área, além da não implementação da política de educação permanente. Esta pesquisa objetivou avaliar as publicações na área quanto as capacitações de recursos humanos e a implantação da educação permanente como estratégia de ensino. Assim, foi realizada uma revisão integrativa por meio de uma busca nas bases de dados Medline e Lilacs no período de fevereiro a março de 2020. O levantamento bibliográfico alcançou uma busca de 516 artigos como amostra inicial, sendo que, após o uso de filtros, permaneceram 38 artigos. Somente 21 foram lidos integralmente, dentre os quais 7 atenderam os critérios de inclusão e exclusão. Identificou-se que existe uma deficiência na política de educação permanente em vigilância sanitária, ausência de projeto político pedagógico, incluindo a temática de Visa nos cursos de ensino superior, falta de investimento e valorização quanto a necessidade de capacitação para os profissionais e, por fim, existe a necessidade de implantar novas formas de aprendizado para os trabalhadores da área. Por ser muito abrangente, esse campo de trabalho exige que os profissionais tenham saberes de diversos campos de atuação para maior efetividade e resolutividade. Faz-se necessária uma intensificação em estratégias educacionais, pois a ausência de uma ampla política de capacitação desses trabalhadores pode causar impactos na proteção da saúde das coletividades.
\end{abstract}

Palavras-chave: Vigilância sanitária, gestão em saúde, estrutura dos serviços, educação continuada.

\section{Health surveillance: integrative review of workforce training processes}

\begin{abstract}
:
Evidence from the Health Surveillance study (Visa) has demonstrated the lack of funding to carry out Visa actions, consequently with few training activities in the area, in addition to the nonimplementation of the permanent education policy. This research aimed to evaluate publications in the area regarding human resources training and the implementation of permanent education as a teaching strategy. Thus, an integrative review was carried out by searching the Medline and Lilacs databases from February to March 2020. The bibliographic survey reached a search for 516 articles as an initial sample, and after using filters, 38 articles and only 21 were read in full, with 7 meeting the inclusion and exclusion criteria. We identified that there is a deficiency in the policy of permanent
\end{abstract}


education in health surveillance, an absence of a Political-Pedagogical Project including the theme of Visa in higher education courses, a lack of investment and appreciation regarding the need for training for professionals and finally there is a need to implement new forms of learning for workers in the field. Because the field of work is very comprehensive, it requires professionals to have knowledge of different fields of activity for greater effectiveness and resolution. It is necessary to intensify educational strategies, as the absence of a broad training policy for these workers can cause impacts in terms of protecting the health of communities.

Keywords: Health surveillance, health management, service structure, continuing education.

\title{
Vigilancia de la salud: revisión integradora de los procesos de capacitación laboral
}

\begin{abstract}
Resumen:
La evidencia del estudio de Vigilancia de la Salud (Visa) ha demostrado la falta de financiamiento para llevar a cabo las acciones de Visa, consecuentemente con pocas acciones de capacitación en el área, además de la no implementación de la política de educación permanente. Esta investigación tuvo como objetivo evaluar publicaciones en el área sobre la formación de recursos humanos y la implementación de la educación permanente como estrategia docente. Así, se realizó una revisión integradora mediante la búsqueda en las bases de datos Medline y Lilacs de febrero a marzo de 2020. La encuesta bibliográfica alcanzó una búsqueda de 516 artículos como muestra inicial, y tras utilizar filtros 38 artículos y solo 21 fueron leídos en su totalidad, con 7 cumpliendo los criterios de inclusión y exclusión. Identificamos que existe una deficiencia en la política de educación permanente en vigilancia de la salud, una ausencia de un Proyecto Político-Pedagógico que incluya la temática de Visa en los cursos de educación superior, una falta de inversión y valoración sobre la necesidad de formación de los profesionales y finalmente existe la necesidad de implementar nuevas formas de aprendizaje para los trabajadores del campo. Debido a que el campo de trabajo es muy amplio, se requiere que los profesionales tengan conocimientos de diferentes campos de actividad para una mayor eficacia y resolución. Es necesario intensificar las estrategias educativas, ya que la ausencia de una política amplia de capacitación para estos trabajadores puede generar impactos en términos de protección de la salud de las comunidades.
\end{abstract}

Palabras clave: vigilancia de la salud, gestión de la salud; estructura de servicio; Educación contínua.

\section{INTRODUÇÃO}

O sistema de saúde no Brasil se consolidou a partir da lei orgânica do Sistema Único de Saúde (SUS), colocando-se como o responsável por proteger a saúde da população através das ações assistenciais ou dos serviços de controle de riscos à coletividade. O SUS se organizou de forma a ser capaz de definir, através de regulamentações específicas, uma forma de permitir o controle no gerenciamento de situações que pudessem comprometer a saúde individual e coletiva (SETA; OLIVEIRA; PEPE, 2017).

As medidas de vigilância não são recentes, entretanto sua sistematização só se consolidou no século XX através da execução, em nível federal, por programas verticalizados capazes de formular e coordenar os sistemas nacionais para a contenção de doenças que 
assolavam o país. Historicamente, a industrialização brasileira só ocorreu efetivamente nos anos 70, resultando numa mudança epidemiológica e demográfica, e exigindo novos saberes e práticas em saúde (FRANCO NETTO et al., 2017).

A criação da Agência Nacional de Vigilância Sanitária (ANVISA) se deu durante o período da reforma do Estado na década de 90, quando foram propostos modelos de agências autônomas. Algumas são reguladoras e outras, executivas com autonomia em relação ao governo federal, englobando as atividades que, antes, eram exclusivamente exercidas pela gestão do Estado, em que a ANVISA fora umas das oito agências criadas no período, demonstrando o caráter de desenvolvimentismo econômico no país (KORNIS; BRAGA; FAGUNDES, 2011).

A agência foi caracterizada pela Lei 9.782 em 1999:

Fica a Agência Nacional de Vigilância Sanitária - ANVISA, autarquia sob regime especial, vinculada ao Ministério da Saúde, com sede e foro no Distrito Federal, prazo de duração indeterminado e atuação em todo território nacional (BRASIL, 1999, p. 1).

O papel da ANVISA, hoje, se institui como um grande gerenciador de riscos, das deficiências ou das adversidades que surgem da utilização de produtos de interesse à saúde. Desse modo, exige grande conhecimento técnico e multidisciplinar dos profissionais que atuam no setor e que convivem rotineiramente com conflitos diversos, seja no campo econômico ou administrativo. O objetivo primordial é a proteção da população (FIGUEIREDO; RECINE; MONTEIRO, 2017).

Para a vigilância, foram definidas competências distintas para as três instâncias de governo, sendo que a execução ficou sob competência municipal em sua totalidade, necessitando superar todos os obstáculos que essas definições de atribuições acarretam (STEINBACH et al., 2012).

Com a descentralização das atividades para o nível local, a coletividade deveria estar mais protegida, já que as ações de Visa deixaram de ser meramente cartoriais. Apesar disso, algumas particularidades surgem e devem ser consideradas para fins de consolidação do sistema de proteção (OLIVEIRA, 2018).

O papel da vigilância sanitária está amparado no âmbito legal, técnico e administrativo, com particularidades para contribuir com a integralidade do cuidado em 
saúde através da qualidade dos produtos, processos, serviços e ambientes a partir da fiscalização e da inspeção sanitária, das atividades de educação em saúde e de alertas sanitários ou pelo acompanhamento do cumprimento das normas, e aplicando sanções ao setor regulado que as descumprem (LEAL; TEIXEIRA, 2017).

O Plano Diretor de Vigilância Sanitária (PDVISA), instituído em 2007, em seu eixo 4, produção do conhecimento, pesquisa e desenvolvimento tecnológico, reconhece que a atuação diária do profissional de Visa deve ser pautada não só no aparato legal e normativo, mas também em um conjunto de atividades relacionadas a produtos, serviços e ambientes. Assim, necessita de estratégias de qualificação, capacitação e formação permanente para desenvolvimento de competência profissional no processo de trabalho em Visa (BRASIL, 2007).

Em 2006, foi realizado por iniciativa do Comitê de Política de Recursos Humanos para a Vigilância Sanitária (COPRH) da ANVISA apoiado pela Organização Pan-Americana da Saúde (OPAS) e com a colaboração do Núcleo de Estudos em Saúde Coletiva da Universidade Federal do Rio Grande do Norte, o primeiro Censo Visa. Nele, constatou-se que, dos 32.135 trabalhadores entrevistados, 32,6\% deles possuíam escolaridade em nível superior, 60,8\% tinham pós-graduação e 44,4\% haviam concluído cursos de atualização no último ano, tendo apontado a necessidades de disponibilização constante de processos de ensino aprendizagem para garantir uma atuação mais segura e efetiva dos fiscais sanitários, e não colocar em risco as atividades que são desenvolvidas (NETO et al., 2018).

A atividade sanitária exige do profissional uma dinâmica muito grande pelo caráter antecipatório que possui, além de ter que acompanhar a rápida evolução da tecnologia, dos novos produtos e serviços que surgem diariamente, necessitando de permanente atualização e capacitação, capaz de atender as necessidades desta atividade (RAMOS et al., 2017, p. 3-4).

Mas, diante do mundo moderno é necessário refletir muito além do papel apenas de regular as atividades econômicas do país com a finalidade de contribuir com o desenvolvimento econômico, necessitando para isso fomentar o diálogo entre os especialistas que não possuem a prática de troca de saberes e que não se consideram parte de um mundo com muitas necessidades de saúde e lutas sociais, se escondendo atrás dos conhecimentos científicos e desconsiderando que o papel universal deve estar muito além da ciência que não 
permite o equilíbrio diante da racionalidade moderna universal que fundamenta o poder regulatório (PORTO, 2017).

A Política Nacional de Educação Permanente em Saúde, instituída em 2004, trouxe em sua concepção a proposta de incorporar o ensino junto com a prática no aprendizado, estimulando reflexões pelos trabalhadores, para que juntos ocorram transformações no dia a dia das atividades desenvolvidas e através da análise crítica possam fazer transformações nas realidades com o objetivo de qualificar as ações promovendo maior eficiência e resolutividade (BRASIL, 2018).

A partir dessa questão, realizou-se uma revisão integrativa objetivando compreender: Qual o cenário encontrado quanto ao processo de qualificação dos profissionais para atuarem na vigilância sanitária?

\section{METODOLOGIA/ MATERIAL E MÉTODOS}

$\mathrm{Na}$ revisão integrativa da literatura, foram seguidos os seguintes passos: a identificação do problema de pesquisa; a busca da literatura através da delimitação com os descritores, a definição das bases de dados, a aplicação dos critérios de inclusão e exclusão para selecionar os artigos a serem trabalhados; finalizando com a avaliação, análise dos dados levantados e discussão.

Para a elaboração da pergunta norteadora, utilizou-se da estratégia PICO (P- populatin; Iintervention; C- comparison; O- outcome) que significam "população", "intervenção", “comparação" e "resultados esperados", respectivamente. Diante disso, a definição da pesquisa foi: população: vigilância sanitária; intervenção: gestão em saúde; comparação: educação continuada; resultados esperados: estrutura dos serviços ofertados, permitindo, assim, a definição da pergunta norteadora.

A busca da literatura foi realizada no período entre fevereiro e março de 2020. Os critérios de inclusão foram artigos em português, inglês e espanhol publicados nos últimos quatro anos que trouxessem considerações sobre o cenário encontrado quanto ao processo 
de qualificação dos profissionais de vigilância sanitária indexado nas bases de dados Lilacs e Medline.

A escolha das bases de dados se deu devido a especificidade da temática está direcionada para a área da gestão na saúde, não sendo tão explorada em conteúdos assistenciais, nas quais outras bases teriam maior abrangência.

No processo de busca, foram feitas combinações entre as seguintes palavras-chaves consideradas descritores no DeCS (Descritores em Ciências da Saúde) e MeSH (Medical Subject Headings): Vigilância Sanitária (Health surveillace); Educação Continuada (Education continuing); Gestão de risco (Risk managment); Estrutura dos serviços (Structure of services); Recursos Humanos (Human resources); Vigilância em Saúde Pública (Public Health Surveillance); Gestão em Saúde (Health Management). Os termos foram cruzados com descritores e, também, com palavras do título ou resumo.

A seleção dos descritores foi realizada com base na maior relação dos elementos chave para contemplar o assunto do trabalho. Destaca-se que há alguns termos, como "educação permanente", que não foram utilizados apesar do seu significado peculiar na pesquisa.

Possibilitamos a identificação de artigos científicos nas bases de dados escolhidas que, após seleção criteriosa, puderam contribuir com as questões da pesquisa, seguindo as possibilidades de rearranjos junto aos descritores utilizando os operadores boleanos $O R$ e AND.

Ao analisar a Tabela 1, na qual são apresentadas as duas bases de dados trabalhadas, as palavras-chave foram cruzadas de seis maneiras distintas na base Lilacs e de sete maneiras distintas na Medline, utilizando os operadores boleanos AND e OR, conforme apresentado. Nas colunas sequenciais, pode-se observar o quantitativo de referências obtidas, que permitiu a seleção para avaliação de alguns resumos e, logo após, a identificação dos artigos que seriam analisados na íntegra. Desse modo, evidenciam-se quais artigos restantes fariam parte da análise substancial do trabalho em questão, sendo demonstrado o quantitativo nas colunas da Tabela 1. 
Tabela 1 - Distribuição das referências bibliográficas nas bases Lilacs e Medline segundo as palavras-chave selecionadas, Brasil, 2020.

\begin{tabular}{|c|c|c|c|c|c|}
\hline $\begin{array}{l}\text { BASE DE } \\
\text { DADOS }\end{array}$ & $\begin{array}{l}\text { PALAVRAS-CHAVE } \\
\text { CRUZADAS } \\
\text { CONCOMITANTEMENTE } \\
\text { (COMO PALAVRAS DO } \\
\text { RESUMO E COMO } \\
\text { DESCRITOR) }\end{array}$ & $\begin{array}{l}\text { NÚMERO DE } \\
\text { REFERÊNCIAS } \\
\text { OBTIDAS }\end{array}$ & $\begin{array}{c}\text { RESUMOS } \\
\text { ANALISADOS }\end{array}$ & $\begin{array}{l}\text { REFERÊNCIAS } \\
\text { SELECIONADAS } \\
\text { PARA ANÁLISE }\end{array}$ & $\begin{array}{l}\text { SELECIONADOS } \\
\text { PARA REVISÃO }\end{array}$ \\
\hline Lilacs & $\begin{array}{l}\text { Vigilância sanitária AND } \\
\text { estrutura dos serviços AND } \\
\text { gerenciamento de risco }\end{array}$ & 0 & 0 & 0 & 0 \\
\hline Lilacs & $\begin{array}{l}\text { Vigilância sanitária AND } \\
\text { estrutura dos serviços OR } \\
\text { recursos humanos }\end{array}$ & 272 & 3 & 2 & 1 \\
\hline Lilacs & $\begin{array}{l}\text { Estrutura dos serviços AND } \\
\text { Recursos humanos AND } \\
\text { Educação continuada }\end{array}$ & 1 & 1 & 0 & 0 \\
\hline Lilacs & $\begin{array}{l}\text { Vigilância em Saúde Pública } \\
\text { OR } \\
\text { Recursos humanos OR } \\
\text { Gerenciamento do risco }\end{array}$ & 443 & 10 & 8 & 3 \\
\hline Lilacs & $\begin{array}{l}\text { Vigilância em Saúde Pública } \\
\text { OR } \\
\text { Gestão em saúde AND } \\
\text { Educação continuada }\end{array}$ & 33 & 4 & 01 & 0 \\
\hline Lilacs & $\begin{array}{l}\text { Vigilância sanitária AND } \\
\text { recursos humanos AND } \\
\text { Gerenciamento do risco }\end{array}$ & 0 & 0 & 0 & 0 \\
\hline Lilacs & $\begin{array}{l}\text { Vigilância sanitária OR } \\
\text { educação continuada OR } \\
\text { Recursos humanos }\end{array}$ & 257 & 4 & 2 & 0 \\
\hline Medline & $\begin{array}{l}\text { Vigilância sanitária AND } \\
\text { estrutura dos serviços AND } \\
\text { gerenciamento de risco AND } \\
\text { gestão do risco }\end{array}$ & 21 & 0 & 0 & 0 \\
\hline Medline & $\begin{array}{l}\text { Vigilância sanitária AND } \\
\text { estrutura dos serviços AND } \\
\text { recursos humanos }\end{array}$ & 58 & 1 & 1 & 0 \\
\hline Medline & $\begin{array}{l}\text { Estrutura dos serviços AND } \\
\text { Recursos humanos AND } \\
\text { Educação continuada }\end{array}$ & 51 & 1 & 1 & 0 \\
\hline Medline & $\begin{array}{l}\text { Vigilância em Saúde Pública } \\
\text { AND } \\
\text { Recursos humanos AND } \\
\text { Gerenciamento do risco }\end{array}$ & 39 & 1 & 1 & 1 \\
\hline Medline & $\begin{array}{l}\text { Vigilância em Saúde Pública } \\
\text { AND } \\
\text { Gestão em saúde AND } \\
\text { Educação continuada }\end{array}$ & 40 & 4 & 3 & 2 \\
\hline Medline & $\begin{array}{l}\text { Vigilância sanitária AND } \\
\text { recursos humanos AND } \\
\text { Gerenciamento do risco }\end{array}$ & 107 & 9 & 0 & 0 \\
\hline Medline & $\begin{array}{l}\text { Vigilância sanitária OR } \\
\text { educação continuada OR } \\
\text { Recursos humanos }\end{array}$ & 01 & 0 & 0 & 0 \\
\hline
\end{tabular}

Fonte: Elaborado pelos autores. 


\section{RESULTADOS}

Os cruzamentos e análises realizadas no estudo possibilitaram a identificação de um total de 516 artigos divididos em 128 artigos científicos na base de dados Lilacs, representando 24,8\% e 388 na Medline (75,2\%). Foram selecionados 38 para a leitura dos resumos. Logo, foram definidos 21 artigos para serem lidos integralmente.

Depois da leitura crítica desses artigos, definiu-se trabalhar somente com sete artigos, conforme demonstrado no Quadro 1. Nele, os artigos estão apresentados para a revisão integrativa, e descritos com o nome da revista de publicação, título do artigo, ano, grau de evidência com o tipo de estudo e as principais conclusões de cada um.

Quadro 1 - Distribuição das referências incluídas na revisão integrativa, de acordo com período publicado, ano da publicação, grau de evidência/ método e conclusão, Brasil, 2020.

\begin{tabular}{|c|c|c|c|c|c|}
\hline $\mathbf{n}$ & PERIÓDICO & TÍTULO & $\begin{array}{c}\text { ANO } \\
\text { PUBLICAÇÃO }\end{array}$ & $\begin{array}{c}\text { GRAU DE } \\
\text { EVIDÊNCIA/TIPO } \\
\text { DE ESTUDO }\end{array}$ & PRINCIPAIS CONCLUSÕES \\
\hline 1 & $\begin{array}{l}\text { Physis } \\
\text { Revista de } \\
\text { Saúde } \\
\text { Coletiva }\end{array}$ & $\begin{array}{l}\text { Avaliação da } \\
\text { organização } \\
\text { estrutural das } \\
\text { vigilâncias } \\
\text { sanitárias } \\
\text { municipais de } \\
\text { uma região de } \\
\text { saúde de } \\
\text { Pernambuco }\end{array}$ & 2017 & 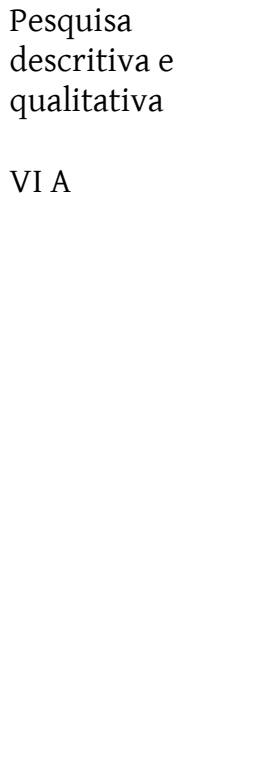 & $\begin{array}{l}\text { O artigo analisou a estrutura } \\
\text { organizacional de Visa de onze } \\
\text { municípios do Estado de } \\
\text { Pernambuco, apontando, pelo } \\
\text { estudo, grande fragilidade nas } \\
\text { estruturas municipais, } \\
\text { dificultando atender as demandas } \\
\text { do território, em que se destaca } \\
\text { grande deficiência na política de } \\
\text { educação permanente para os } \\
\text { trabalhadores de Visa. Demonstra } \\
\text { a necessidade de engajamento dos } \\
\text { gestores para garantir a estrutura } \\
\text { adequada e capacidade } \\
\text { operacional com educação } \\
\text { contínua dos trabalhadores para } \\
\text { que as demandas territoriais } \\
\text { possam ser atendidas } \\
\text { satisfatoriamente. }\end{array}$ \\
\hline 2 & $\begin{array}{l}\text { Revista } \\
\text { Escola de } \\
\text { Enfermagem } \\
\text { USP }\end{array}$ & $\begin{array}{l}\text { O ensino de } \\
\text { vigilância } \\
\text { sanitária na } \\
\text { formação do } \\
\text { enfermeiro }\end{array}$ & 2017 & $\begin{array}{l}\text { Estudo } \\
\text { exploratório, com } \\
\text { métodos mistos, } \\
\text { tanto de dados } \\
\text { quantitativos } \\
\text { quanto de } \\
\text { qualitativos } \\
\text { VI A }\end{array}$ & $\begin{array}{l}\text { O estudo apontou que a maioria dos } \\
\text { projetos político pedagógicos nas } \\
\text { faculdades de Enfermagem não } \\
\text { contemplam a disciplina de Visa, e } \\
\text { que a categoria do profissional } \\
\text { enfermeiro é a segunda categoria } \\
\text { que mais exerce atividades nessa } \\
\text { área, levando os profissionais a } \\
\text { recorrerem a muitos cursos de } \\
\text { especialização após a conclusão do }\end{array}$ \\
\hline
\end{tabular}




\begin{tabular}{|c|c|c|c|c|c|}
\hline & & & & & $\begin{array}{l}\text { ensino superior. A ANVISA também } \\
\text { indicou que a área de Visa possui } \\
\text { deficiência quanto a capacitação } \\
\text { dos profissionais em atuação na } \\
\text { área, apontando alternativas para } \\
\text { superar esse problema. }\end{array}$ \\
\hline 3 & $\begin{array}{l}\text { Revista de } \\
\text { Saúde } \\
\text { Coletiva }\end{array}$ & $\begin{array}{l}\text { Translação do } \\
\text { Conhecimento } \\
\text { na } \\
\text { qualificação } \\
\text { da gestão da } \\
\text { Vigilância em } \\
\text { Saúde: } \\
\text { contribuição } \\
\text { dos estudos } \\
\text { avaliativos de } \\
\text { pós-graduação }\end{array}$ & 2019 & $\begin{array}{l}\text { Estudo de caso, } \\
\text { qualitativo } \\
\text { VI A }\end{array}$ & $\begin{array}{l}\text { O estudo apontou que a translação } \\
\text { do conhecimento na qualificação } \\
\text { da gestão da Vigilância em Saúde, } \\
\text { estando aqui incluída a Visa, deu-se } \\
\text { em todas as dimensões, ocorrendo } \\
\text { maior destaque a nível individual e } \\
\text { interpessoal, em que os } \\
\text { pesquisadores conseguiram } \\
\text { favorecer a aplicação do } \\
\text { conhecimento. }\end{array}$ \\
\hline 4 & $\begin{array}{l}\text { Espaço para } \\
\text { a saúde - } \\
\text { Revista de } \\
\text { Saúde } \\
\text { Pública do } \\
\text { Paraná }\end{array}$ & $\begin{array}{l}\text { Vigilância em } \\
\text { saúde no } \\
\text { âmbito } \\
\text { municipal: } \\
\text { revisão } \\
\text { integrativa }\end{array}$ & 2017 & $\begin{array}{l}\text { Revisão } \\
\text { integrativa de } \\
\text { literatura } \\
\text { VIA }\end{array}$ & $\begin{array}{l}\text { A revisão integrativa foi capaz de } \\
\text { apresentar o conceito ampliado de } \\
\text { Vigilância em Saúde, apresentando } \\
\text { o processo de municipalização e os } \\
\text { setores que compõe o processo de } \\
\text { trabalho da vigilância, incluindo a } \\
\text { sanitária, apresentando os } \\
\text { rearranjos municipais para } \\
\text { execução as atividades preventivas } \\
\text { dentro da esfera municipal. }\end{array}$ \\
\hline 5 & $\begin{array}{l}\text { Revista } \\
\text { Ciência e } \\
\text { Saúde } \\
\text { Coletiva }\end{array}$ & $\begin{array}{l}\text { Residência } \\
\text { Integrada em } \\
\text { Saúde com } \\
\text { ênfase em } \\
\text { vigilância } \\
\text { sanitária }\end{array}$ & 2017 & $\begin{array}{l}\text { Descritivo de } \\
\text { relato de } \\
\text { experiência. } \\
\text { VIA }\end{array}$ & $\begin{array}{l}\text { O relato de experiência descrito no } \\
\text { trabalho mostra o aprendizado } \\
\text { alcançado pelos egressos ao } \\
\text { retornarem ao mercado de } \\
\text { trabalho, seja trabalhando } \\
\text { diretamente no SUS seja } \\
\text { aproveitando a experiência em } \\
\text { outras atividades. Através da } \\
\text { Residência Integrada em Saúde } \\
\text { com ênfase em Visa, é permitido } \\
\text { conhecer a profundidade dos } \\
\text { trabalhados executados e novos } \\
\text { olhares para as realidades } \\
\text { existentes, e promover cada vez } \\
\text { mais um SUS integral e igualitário. }\end{array}$ \\
\hline 6 & $\begin{array}{l}\text { Revista de } \\
\text { Saúde } \\
\text { Pública }\end{array}$ & $\begin{array}{l}\text { Controle } \\
\text { sanitário em } \\
\text { serviços de } \\
\text { saúde: uma } \\
\text { dimensão da } \\
\text { universalidade }\end{array}$ & 2017 & $\begin{array}{l}\text { Ensaio } \\
\text { IV A }\end{array}$ & $\begin{array}{l}\text { A reflexão do estudo demonstrou a } \\
\text { necessidade que a Visa carece para } \\
\text { poder desempenhar o seu papel de } \\
\text { proteção à sociedade com a } \\
\text { qualidade que é exigida, pela } \\
\text { precariedade quanto aos recursos } \\
\text { humanos, falta de integração de } \\
\text { saberes capazes de contribuir com } \\
\text { a melhoria do trabalho e a falta de } \\
\text { financiamento para implementar } \\
\text { um plano regional de educação } \\
\text { permanente para o serviço de } \\
\text { vigilância sanitária. }\end{array}$ \\
\hline
\end{tabular}




\begin{tabular}{|l|l|l|l|l|l|}
\hline 7 & $\begin{array}{l}\text { Revista } \\
\text { Ciência e } \\
\text { Saúde } \\
\text { Coletiva }\end{array}$ & $\begin{array}{l}\text { Caminhos } \\
\text { possíveis para } \\
\text { a avaliação } \\
\text { das práticas } \\
\text { da Vigilância } \\
\text { em Saúde }\end{array}$ & 2017 & $\begin{array}{l}\text { Pesquisa } \\
\text { avaliativa, } \\
\text { qualitativa } \\
\text { VI A }\end{array}$ & $\begin{array}{l}\text { O estudo propõe, através da } \\
\text { autoavaliação pelas equipes de } \\
\text { Vigilância em Saúde do município } \\
\text { de Guarulhos (SP), um instrumento } \\
\text { positivo para apontar os nós } \\
\text { críticos dos processos de trabalho } \\
\text { da área com seus setores } \\
\text { envolvidos Epidemiologia e } \\
\text { Sanitária. No que tange ao aspecto } \\
\text { de capacitação profissional da } \\
\text { equipe de Visa, constatou-se que } \\
\text { existe uma deficiência, } \\
\text { principalmente pela oferta } \\
\text { insuficiente de vagas para todos os } \\
\text { trabalhadores da área, refletindo } \\
\text { diretamente no processo de } \\
\text { qualificação da atividade diária. }\end{array}$ \\
& & &
\end{tabular}

Fonte: Elaborado pelos autores.

A partir da seleção das publicações, essas foram categorizadas em: 1) Deficiência na política de educação permanente em vigilância: periódicos 1, 4 e 6;2) Ausência de projeto político pedagógico incluindo a temática de vigilância sanitária: periódicos 2 e 5;3) Falta de investimento e valorização quanto a necessidade de capacitação dos profissionais de Visa: periódico 7; 4) Necessidade de implantar novas formas de aprendizado com os profissionais: periódico 3.

Ao discutir a deficiência na política de educação permanente em vigilância sanitária, observa-se que a descentralização das ações trouxe uma grande carga de trabalho para os municípios. Novos conhecimentos sobre as áreas a serem inspecionadas são necessários, assim como o acompanhamento de uma política de educação permanente em Visa através da qual fossem possíveis discussões acerca da conjuntura estrutural e organizacional e práticas de trabalho que contribuíssem com uma atuação mais efetiva (MEDEIROS; MACHADO; PESSOA JÚNIOR, 2017).

Um grande desafio surge ao fazer o link da Visa no seu papel de garantir saúde econômica, sustentabilidade dos estabelecimentos que fiscaliza, além de proteger a sociedade. Para alcançar esse intento, é necessária uma equipe de profissionais em número suficiente e com conhecimentos variados e complementares para que possa atender ao mercado em constante evolução, tanto de tecnologias, quanto de novas atividades que a cada momento surgem. E, ainda, que se priorize o investimento e implantação de uma política regional às novas demandas. 
No intento de se obter uma vigilância em saúde de dimensões ampliadas, a Visa se faz presente, sendo capaz de contribuir com processos de trabalho produtivos de acordo com os problemas demandados pela situação de saúde dos territórios. Para tanto, é essencial uma requalificação em vigilância que permita a complementariedade de conhecimentos para um crescimento mútuo em equipe a fim de atender a nova dimensão de trabalho com articulações.

Quando se pensa em projeto político pedagógico incluindo a temática de vigilância sanitária, evidencia-se que a Visa tem sido considerada a parte mais complexa da Saúde Pública hoje. Isso porque o universo que engloba é extenso por incluir uma vasta gama de normatizações para o controle sanitário da produção, circulação, guarda, transporte e comercialização de substâncias e produtos de interesse para a saúde e controle sanitário de tecnologias médicas, portos, aeroportos e fronteiras e de serviços direta ou indiretamente relacionados com a saúde e o meio ambiente, incluindo a saúde do trabalhador, conhecimentos da economia, jurídico-político e médico-sanitário. Dessa forma, aponta-se para a necessidade da inclusão nos programas de cursos universitários visto que muitos profissionais acabam trabalhando nessa área sem o conhecimento necessário para atuação (LÔBO et al., 2018). A residência integrada com ênfase em vigilância em saúde se coloca como uma proposta promissora por trazer experiência de grande valor, unindo ensino e serviço, e gerando um aprofundamento nas temáticas que incluem a vigilância sanitária. Proporciona ao residente, desse modo, a vivência do olhar sanitário quanto as não conformidades e as possibilidades de avanço nos serviços, contribuindo com atividades práticas de preenchimento de termos de notificação, auto de infração, avaliação de documentações e liberação de alvarás sanitários (ROCHA et al., 2017).

Concernente à falta de investimento e valorização da necessidade de capacitações para profissionais de vigilância sanitária, destaca-se o grande desafio que é colocado para a Visa em seu papel de focar no território através de uma reorganização de atividades rotineiras. Sem o financiamento proporcional, a integralidade das ações pela equipe, que tem seus conhecimentos restritos às normativas, é dificultada (RECKTENWALDT; JUNGES, 2017).

A vigilância sanitária tem sido, muitas vezes, avaliada apenas pela questão de realizar percentual de inspeções sanitárias por programas federais sem levar em conta o processo formativo da equipe e opinião dos sujeitos como forma de entender o porquê de valorizar o 
aprendizado e identificar a fragilidade mais evidente nos profissionais. Por isso, precisa-se ouvir esses trabalhadores e compreender onde seu trabalho é capaz de contribuir com as redes de atenção à saúde (VILELA; SANTOS; KEMP, 2017).

Ao avaliar sobre a importância de implantação de novas formas de aprendizado com os profissionais, destaca-se a necessidade de criação de novas agendas, elaboração de protocolos, mudança do processo de trabalho, instituição de novos fluxos e contratação de novos técnicos com o objetivo de transformar conhecimento em ação, ou mesmo ser capaz de compartilhar conhecimentos. São indispensáveis as etapas de como utilizar, difundir, implementar e transferir saberes, que se resumem em utilizar o aprendido e ser capaz de produzir trabalho em saúde (BEZERRA et al., 2019).

\section{DISCUSSÃO/ ANÁLISE DE DADOS}

O processo de globalização e a importância de uma vigilância atenta e oportuna trouxeram novas discussões na temática da qualificação da força de trabalho em vigilância sanitária, pois, para que os interesses da coletividade e das comunidades sejam preservados, é necessário um Estado representado por um serviço qualificado.

A formação de profissionais de Visa ocorre sem uma sistematização e de forma, muitas vezes, descontínua, precária de conhecimento técnico e ineficiente de ação devido ao isolamento institucional com o ambiente da saúde coletiva sem compreender o papel como uma ação de saúde (GARIBOTTI; HENNINGTON; SELLI, 2006). Por isso, o destaque é dado à inclusão no Projeto Político Pedagógicos da disciplina no campo da vigilância sanitária para que o profissional alcance uma formação menos fragmentada e desarticulada da realidade, tentando favorecer a experiência da interdisciplinaridade que pode ser fundamental para o futuro do trabalhador (LÔBO et al., 2018).

Propostas como a Residência Integrada em Saúde com ênfase em vigilância apresentada no estudo são alternativas capazes de proporcionar, através do ensino versus serviço em saúde, reflexões quanto as realidades consolidando o SUS, proporcionando o 
retorno dos profissionais para o ambiente de trabalho com visões reconstruídas em ambiente multiprofissional (ROCHA et al., 2017).

Ao trabalhar com objetos complexos, a vigilância se caracteriza entre meios da ciência, da saúde e do mercado, necessitando incorporar saberes interdisciplinares que sejam capazes de dar conta do universo que atua diretamente. Dentre eles, medicamentos, alimentos, tecnologias médicas, serviços de interesse da saúde e outros, sendo que todos esses objetos de trabalho são dinâmicos e em constante evolução, principalmente devido à rapidez da ciência nos tempos atuais (SOUZA; COSTA, 2010).

Os estudos acabam por apontar que é deficiente a política de educação permanente mesmo nos dias de hoje apesar do apontamento já, em 2005, pelo PDVISA, representando um papel político, estratégico e pedagógico extremamente importante, e reafirmando o papel da Visa no campo da Saúde Pública. No mesmo ano, o Regulamento Sanitário Internacional (RSI) demonstrou que era necessário um rearranjo mundial capaz de atender as demandas sanitárias futuras, como a que se vivencia contemporaneamente.

\section{CONSIDERAÇÕES FINAIS}

Este estudo apresenta os cenários encontrados quanto aos processos de ensino e aprendizado junto aos profissionais que atuam na vigilância sanitária em espaços locais a fim de contribuir com a Saúde Pública na proteção das coletividades.

A política de educação permanente não foi efetivamente implementada na área da Visa, além das capacitações não considerarem a importância das diversidades de saberes necessários. A falta de uma política educacional de inclusão da temática nos projetos pedagógicos das instituições aponta por um caminho que necessita de avanços.

Somando-se à falta de sensibilização dos gestores quanto ao investimento no campo dos saberes em Visa e implementação da educação permanente em vigilância sanitária para garantir um serviço que contribui com a integralidade das ações de saúde no SUS, constatase que, no geral, ainda há escassez de estudos com esse tema. 


\section{REFERÊNCIAS}

BEZERRA, L. C. A.; FELISBERTO, E.; COSTA, J. M. B. S.; HARTZ, Z. Translação do Conhecimento na qualificação da gestão da Vigilância em Saúde: contribuição dos estudos avaliativos de pós-graduação. Physis: Revista de Saúde Coletiva, v. 29, n. 1, p. e290112, 2019. Disponível em: <https://www.epublicacoes.uerj.br/index.php/physis/article/view/43298>. Acesso em: 04 set. 2020.

BRASIL. Sistema Nacional de Vigilância Sanitária. Lei № 9.782, de 26 de janeiro de 1999. Disponível em: <http://www.planalto.gov.br/ccivil_03/leis/19782.htm>

<http://www.planalto.gov.br/ccivil_03/leis/19782.htm>. Acesso em: 1 mar. 2020.

BRASIL. Ministério da Saúde. Política Nacional de Educação Permanente em Saúde: o que se tem produzido para o seu fortalecimento? Brasília, 2018. Disponível em: <https://bvsms.saude.gov.br/bvs/publicacoes/politica_nacional_educacao_permanente_saude_fortaleciment o.pdf>. Acesso em: 4 set. 2020.

FIGUEIREDO, A. V. A.; RECINE, E.; MONTEIRO, R. Regulação dos riscos dos alimentos: as tensões da Vigilância Sanitária no Brasil. Ciência \& Saúde Coletiva, v. 22, n. 7, p. 2353-2366, jul. 2017. Disponível em: <https://www.scielo.br/scielo.php?pid=\$1413-81232017002702353\&script=sci_abstract\&tlng=pt>. Acesso em: 04 set. 2020.

FRANCO NETTO, G.; VILLARDI, J. W. R.; MACHADO, J. M. H.; SOUZA, M. S.; BRITO, I. F.; SANTORUM, J. A.; REIS, C. O. O.; FENNER, A. L. D. Vigilância em Saúde brasileira: reflexões e contribuição ao debate da 1a Conferência Nacional de Vigilância em Saúde. Ciência \& Saúde Coletiva, v. 22, n. 10, p. 3137-3148, out. 2017. Disponível em: <https://www.scielo.br/scielo.php?pid=S1413-81232017021003137\&script=sci_abstract\&tlng=pt>. Acesso em: 04 set. 2020.

GARIBOTTI, V.; HENNINGTON, É. A.; SELLI, L. A contribuição dos trabalhadores na consolidação dos serviços municipais de vigilância sanitária. Cadernos de Saúde Pública, v. 22, n. 5, p. 1043-1051, maio 2006. Disponível em: <https://www.scielo.br/scielo.php?script=sci_arttext\&pid=S0102-311X2006000500017>. Acesso em: 04 set. 2020.

KORNIS, G. E. M.; BRAGA, M. H.; FAGUNDES, M. A regulação em saúde no Brasil: um breve exame das décadas de 1999 a 2008. Physis Revista de Saúde Coletiva, v. 21, n. 3, p.1077-1102, 2011. Disponível em: <https://www.scielo.br/scielo.php?script=sci_arttext\&pid=S0103-73312011000300017>. Acesso em: 04 set. 2020.

LEAL, C. O. B. S.; TEIXEIRA, C. F. DE S. Solidariedade: uma perspectiva inovadora na gestão e organização das ações de Vigilância Sanitária. Ciência \& Saúde Coletiva, v. 22, n. 10, p. 3161-3172, out. 2017. Disponível em: <https://www.scielo.br/scielo.php?pid=S1413-81232017021003161\&script=sci_abstract\&tlng=pt>. Acesso em: 04 set. 2020.

LÔBO, C. D. de A.; CUNHA, M. C. S. O; PONTE, V. A; COSTA, E. C; ARAÚJO, M. F. A; ARAÚJO, T. M. O ensino de vigilância sanitária na formação do enfermeiro. Revista da Escola de Enfermagem da USP, v. 52, n. 0, 13 dez. 2018. Disponível em: <https://www.scielo.br/pdf/reeusp/v52/1980-220X-reeusp-52-e03387.pdf>. Acesso em: 04 set. 2020.

MEDEIROS, E. H. F. de; MACHADO, M. F.; PESSOA JÚNIOR, J. M. Avaliação da organização estrutural das vigilâncias sanitárias municipais de uma Região de Saúde de Pernambuco. Physis: Revista de Saúde Coletiva, v. 27, n. 3, p. 561-578, jul. 2017. Disponível em: <https://www.scielo.br/scielo.php?pid=S0103$73312017000300561 \&$ script=sci_abstract\&tlng=pt>. Acesso em: 04 set. 2020.

NETO, M.; GODIM, A.; BATISTA, J.; LOPES, N. Vigilância Sanitária: a necessidade de reorientar o trabalho e a qualificação em um município. Vigilância Sanitária em Debate, v. 6, p. 56, 30 nov. 2018. Disponível em: <https://pesquisa.bvsalud.org/portal/resource/pt/biblio-966785>. Acesso em: 04 set. 2020. 
OLIVEIRA, A. M. C. Caminhos para a Vigilância Sanitária: o desafio da fiscalização nos serviços de saúde. Visa em $\begin{array}{lllllll}\text { Debate, } & \text { v. } & 6 & \text { n. } & 3, & 2018 . & \text { Disponível }\end{array}$ <https://visaemdebate.incqs.fiocruz.br/index.php/visaemdebate/article/view/1114>. Acesso em: 04 set. 2020.

PORTO, M. F. DE S. Pode a Vigilância em Saúde ser emancipatória? Um pensamento alternativo de alternativas em tempos de crise. Ciência \& Saúde Coletiva, v. 22, n. 10, p. 3149-3159, out. 2017. Disponível em: <https://www.scielo.br/scielo.php?pid=\$1413-81232017021003149\&script=sci_abstract\&tlng=pt>. Acesso em: 04 set. 2020.

RAMOS, L.; PETTA, H. L.; MASSARO, A.; LIMA, V. V.; ARAÚJO, D. G.; BRITO, M. C. M.; GOMES, R. Nota técnica: perfil da competência do especialista em Gestão da Vigilância Sanitária: no 1.1. São Paulo: Instituto SírioLibanês de Ensino e $2017 . \quad$ Desquisa, <http://iep.hospitalsiriolibanes.org.br/Documents/LatoSensu/NotaTecnica1.1.pdf>. Acesso em: 04 set. 2020.

RECKTENWALDT, M.; JUNGES, J. R. A organização e a prática da Vigilância em Saúde em municípios de pequeno porte. Saúde e Sociedade, v. 26, n. 2, p. 367-381, jun. 2017. Disponível em: <https://www.scielo.br/scielo.php?script=sci_arttext\&pid=S0104-12902017000200367>. Acesso em: 04 set. 2020.

ROCHA, A. F. da; BREIER, A.; SOUZA, B.; ALMEIDA, C. N.; SANTOS, C. M.; ROHLOFF, C. C.; CANAL, N.; SANTOS, N. M.; REINHER, S. G. M. Sobre a Residência Integrada em Saúde com ênfase em Vigilância em Saúde. Ciência \& Saúde Coletiva, v. 22, n. 10, p. 3467-3472, out. 2017. Disponível em: <https://www.scielo.br/scielo.php?pid=S1413-81232017021003467\&script=sci_abstract\&tlng=pt>. Acesso em: 04 set. 2020.

SETA, M. H. D.; OLIVEIRA, C. V. dos S.; PEPE, V. L. E. Proteção à saúde no Brasil: o Sistema Nacional de Vigilância Sanitária. Ciência \& Saúde Coletiva, v. 22, n. 10, p. 3225-3234, out. 2017. Disponível em: <https://www.scielo.br/scielo.php?pid=S1413-81232017021003225\&script=sci_abstract\&tlng=pt>. Acesso em: 04 set. 2020.

SOUZA, G. S.; COSTA, E. A. Considerações teóricas e conceituais acerca do trabalho em vigilância sanitária, campo específico do trabalho em saúde. Ciência \& Saúde Coletiva, v. 15, n. suppl 3, p. 3329-3340, nov. 2010. Disponível em: <https://www.scielo.br/scielo.php?script=sci_arttext\&pid=S1413-81232010000900008>. Acesso em: 04 set. 2020 .

STEINBACH, A.; SCHWENGBER, L. M. K; MARTINS, C.; DUTRA, A.; VENÂNCIA, D.; SILVA, M. Z. Mapeamento da produção científica em gestão da vigilância sanitária no período 2000 a 2010. Revista Eletronica Gestão \& Saúde, v. 3, n. 3, p. 641-662, 2012. Disponível em:<https://periodicos.unb.br/index.php/rgs/article/view/130>. Acesso em: 04 set. 2020.

VILELA, M. F. de G.; SANTOS, D. N. dos; KEMP, B. Caminhos possíveis para a avaliação das práticas da Vigilância em Saúde. Ciência \& Saúde Coletiva, v. 22, n. 10, p. 3183-3192, out. 2017. Disponível em: <https://www.scielo.br/scielo.php?pid=\$1413-81232017021003183\&script=sci_abstract\&tlng=pt>. Acesso em: 04 set. 2020.

\section{(c) $)_{\text {EY }}$}

Este trabalho está licenciado com uma Licença Creative Commons - Atribuição 4.0 Internacional. 